\title{
Haemofiltration and Noradrenaline Corrects life-Threatening Cardiovascular Instability Following Delayed Anaphylactic Reaction to Patent Blue Dye in Sentinel Node Biopsy for Breast Cancer
}

\author{
Peter K. Donnelly¹, Louise Robinson², Matt Halkes², Andy Goodman³ \\ ${ }^{1}$ Department of Breast Surgery, Torbay Hospital, South Devon Healthcare NHS Foundation Trust, Torquay, UK \\ ${ }^{2}$ Department of Anaesthesia, Torbay Hospital, South Devon Healthcare NHS Foundation Trust, Torquay, UK \\ ${ }^{3}$ Department of Oncology, Torbay Hospital, South Devon Healthcare NHS Foundation Trust, Torquay, UK \\ Email: peter.donnelly@nhs.net
}

Received 20 August 2014; revised 15 September 2014; accepted 9 October 2014

Copyright @ 2014 by authors and Scientific Research Publishing Inc.

This work is licensed under the Creative Commons Attribution International License (CC BY). http://creativecommons.org/licenses/by/4.0/

\section{(c) (i) Open Access}

\section{Abstract}

The aim of this case report is to present a novel approach to the management of very severe anaphylactic reaction to Patent Blue Dye used in Sentinel Node Biopsy for breast cancer treatment. The severity and duration of cardiovascular effects has not been described previously in this setting. Comprehensive ICU support including heamofiltration resulted in the patients' complete recovery and successful long-term well-being despite a change to our routine cancer care plan.

\section{Keywords}

Blue Dye, Anaphylaxis, Sentinel Node, Haemofiltration, Breast Cancer

\section{Introduction}

Sentinel node biopsy with dual localization using blue dye and radioisotope is the standard of care for early breast cancer treatment, this being the preferred method for staging the axilla in early breast cancer in the United Kingdom (NICE Guidelines 2009 [1]).

Although severe allergic reaction to blue dye has been described there are, in the sentinel node era, no reported deaths or patient needing ventilation beyond 24 hours and no alteration to the recommended cancer plan 
[2]-[4]. We have reported a Grade 3 anaphylactic reaction with a near-death consequence resulting in the need to give prolonged vasopressor therapy, haemofiltration and a significant change to the recommended cancer care plan.

\section{Case Report}

As a result of screening mammography, a 59-year-old woman was found to have a $10 \mathrm{~mm}$ invasive ductal carcinoma of her left breast. Ultrasound of the axilla was normal. She had a past history of palpitations treated by propranolol $40 \mathrm{mg}$ daily and was otherwise well with no known allergies. Thirty years earlier she had an uneventful general anaesthetic for tubal insufflations using methylene blue.

The patient was admitted for breast wide local excision with ultrasound wire and sentinel node biopsy having previously given consent and provided with information in line with NICE [1] and NEW START [2] guidance. Periareolar injection of 20 MBq of radioactively labelled colloid Tc99m (1.25 ml; Nanocoll; Amersham Healthcare Ltd., Buckinghamshire, UK) was administered 3 hour before the operation. Pre-operatively lymphosintigraphy identified three hot lymph glands in the left axilla

General anaesthesia was induced with Fentanyl, Propofol and Atracurium,Co-amoxiclav $1200 \mathrm{mg}$ was given intravenously as antibiotic prophylaxis. The airway was secured with an endotracheal tube and positive pressure ventilation commenced. The anaesthetist was alerted to the injection by the surgeon of 2 mls of Patent Blue V (Patent Blue V Sodium Guerbet 2.5\%) intra-dermally in the left breast peri-areola skin. Forty minutes after induction the non-invasive blood pressure became un-recordable. Boluses of initially Ephedrine and then Metaraminol were administered and technical issues with the blood pressure cuff excluded. A pre-auricular pulse remained palpable, but a non-invasive pressure remained un-recordable. At this time the surgeon noted oedema of the surgical field and examination of the patient revealed facial swelling. A presumptive diagnosis of anaphylaxis was made and $50 \mathrm{mcg}$ of adrenaline administered intravenously. Invasive arterial monitoring was established and confirmed hypotension (systolic $60 \mathrm{mmHg}$ ). Further aliquots of adrenaline were administered with adequate, but transient effect and an adrenaline infusion commenced. Intravenous Chlorphenamine and Dexamethasone were also given as second line therapies. The patient's heart rate remained stable at 50 - $60 \mathrm{bpm}$ throughout 15 minutes of hypotension.

The surgery was speedily concluded and the patient was transferred to ICU for ongoing supportive care. She exhibited further episodes of cardiovascular instability which were managed with fluid challenges and further increments of the adrenaline infusion (peak dose $0.33 \mathrm{mcg} / \mathrm{kg} / \mathrm{min}$ ). The patient did develop widespread angioedema (Figure 1 and Figure 2), but did not develop a rash or bronchospasm. Biphasic blue skin discolouration consistent with leaching of dye from the injection site was noted during the first 48 hrs after surgery.

Fifteen hours after admission the patient developed a significant metabolic acidosis (Base excess -12.9 $\mathrm{mmol} / \mathrm{L}$ and lactate 9.4). At this time noradrenaline was substituted for the adrenaline infusion and continuous venous-venous haemo-filtration commenced. The aim of the latter was not only to address the metabolic acidosis, but also to promote clearance of the patent blue dye as the clinical picture was of episodic cardio vascular instability suggestive of ongoing antigenic stimulus (Figure 3). On this basis CVVHD was continued for twenty four hours in total although the lactic acidosis resolved within eight hours. Noradrenaline requirements peaked on the second day of admission at $0.71 \mathrm{mcg} / \mathrm{kg} / \mathrm{min}$, but then they successfully weaned over the next 72 hours.

An electrocardiograph on admission showed changes suggestive of a potential septalinfarct, but troponin assay proved normal and although an initial echocardiogram demonstrated regional wall abnormalities these were felt to be rate related as they resolved on a repeat echocardiogram.

Additionally a degree of cardiac ischaemia is a consistent finding in acute anaphylaxis due to a combination of coronary artery spasm and systemic hypotension.

Forty eight hours after admission the patient was extubated, but had to be re-intubated 18 hours later due to a combination of progressive hypoxia and increased work of breathing that failed to improve with a trial of non-invasive ventilation. A chest x-ray taken at this time demonstrated bilateral pulmonary interstitial oedema consistent with adult respiratory distress syndrome. A protective lung ventilation strategy was adopted and the patient was able to be successfully extubated 48 hours later, but did require non-invasive ventilatory support. This was weaned over the subsequent 5 days and the patient discharged to ward based care on Day 14 profoundly weakened despite parenteral nutrition.

The patient was discharged to a community hospital on Day 22 and home on Day 26 with a package of support. 


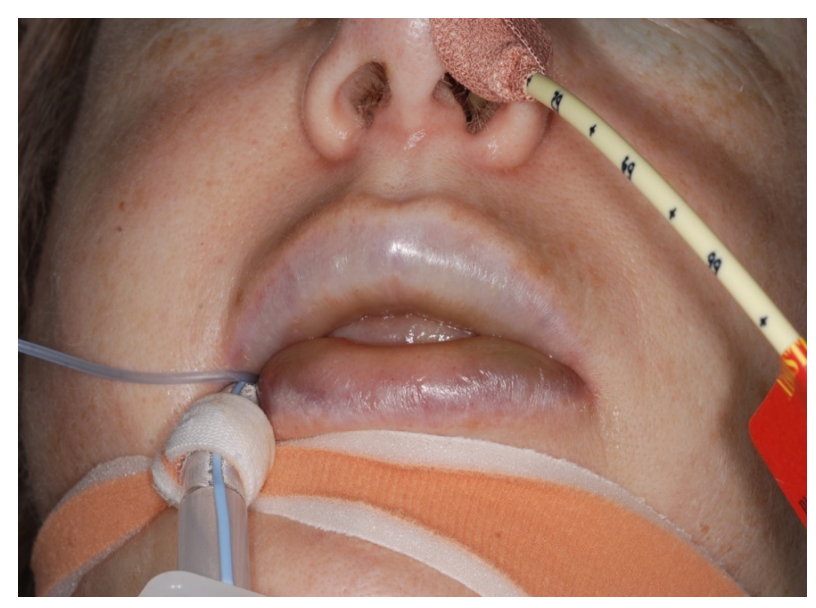

Figure 1. Photograph of intubated patient in ICU, to illustrate angioedema of lips.

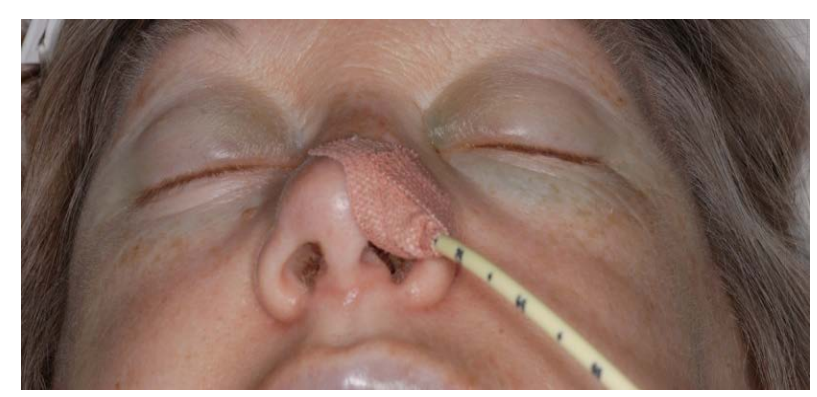

Figure 2. Photograph of patient with naso-gastric feeding in ICU, to illustrate peri-orbital oedema and blue skin discoloration from Patent Blue Dye reaction.

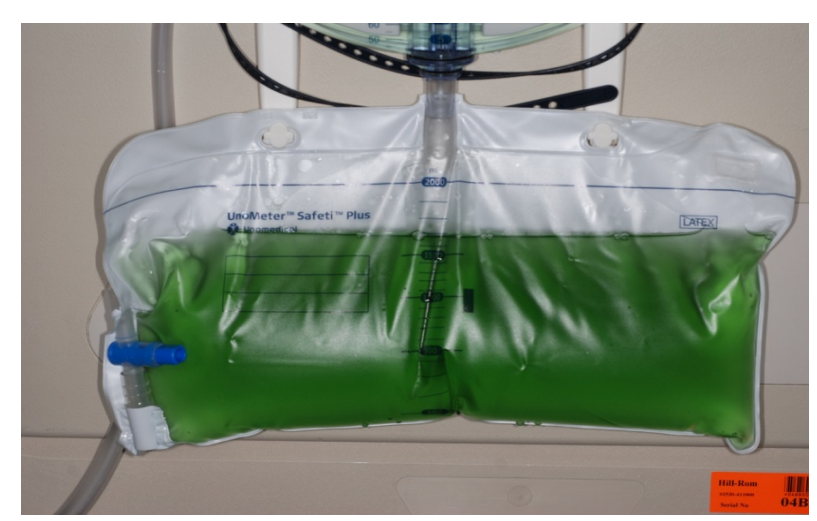

Figure 3. Photograph to illustrate dilute Blue Dye recovered from body fluids during treatment in ICU.

Follow up investigations revealed a peak Tryptase level of $87.9 \mathrm{ng} / \mathrm{ml}$ (normal range 2 - $14 \mathrm{ng} / \mathrm{ml}$ ) and positive skin prick tests (SPTs) to neat 2.5\% PB V. SPTs to all other peri-operative drugs, latex and surgical antiseptics were negative. IgE to latex, penicillin $\mathrm{V}$, penicillin $\mathrm{G}$ and amoxicillin were all negative. A subsequent oral course of Amoxicillin and Flucloxacillin for a chest infection was well tolerated.

Post-operative histology revealed that the breast tumour was a $10 \mathrm{~mm}$ Grade 2 ductal carcinoma with clear excision margins of a minimum of $15 \mathrm{~mm}$ circumferentially and $1 \mathrm{~mm}$ deeply at the pectoral fascia. The tumour was receptor positive for oestrogen, negative for progesterone and HER2. There was no lymphovascular invasion but one of four sentinel nodes showed evidence of macro metastases. 
The patient's management was discussed at a Multidisciplinary Team meeting and with the family 10 days post-operatively and it was agreed that the patient would be unfit for further axillary gland clearance within two weeks. It was agreed that she would have radiotherapy to the breast and axilla and she was given $40 \mathrm{~Gy}$ in 15 treatments to the left breast and nodal areas followed by a boost of $10 \mathrm{~Gy}$ in 5 treatments to the primary site. Whilst adjuvant chemotherapy would also have been routinely discussed this was considered too hazardous as the patient was still on ICU. The patient and family accepted a recommendation for 5 years of hormone blocking treatment and were prescribed Letrazole in a dose of $2.5 \mathrm{mg}$ with calcium supplementation.

Over the next six months the patient recovered well, achieving full mobility but had a persistent dry cough. Chest X-ray 8 months post-operatively showed two ill-defined opacities in the left upper zone but CT scan showed no abnormality. A diagnosis of radiation pneumonitis was made and the patient recovered following a short course of oral steroids. Four years post operatively the patient remains well, with no signs of recurrence clinically or on surveillance mammography.

\section{Discussion}

Blue dye, with or without isotope, is now commonly used to identify sentinel lymph nodes in breast cancer and some other malignancies, e.g. melanoma surgery and in neurosurgery. There was estimated total allergic potential of $0.86 \%$ (anaphylaxis grades 1 - 3) from the combined data sets of the ALMANAC trial and those patients who participated in the NEW START [1]. For severe Grade 3 anaphylaxis, a reaction rate of $0.06 \%$ is reported [4]. Higher rates of $0.4 \%-1.0 \%$ have been reported in other series [5] [6]. These figures may understate the range of responses seen by anaesthetists. Grade 3 anaphylaxis is defined as reaction causing "severe hypotension requiring vasopressor support and/or change/abandoning of planned procedure and/or HDU/ITU admission” [4].

Patent Blue V (PB V) dye has been used since the 1960's in lymphangiography and outside medicine is used in textiles and as a food colourant E131. This more widespread use potentially exposes the general population to the risk of allergic sensitization [7] [8].

Anaphylaxis to anaesthetic drugs is variably described to be of the order of 1:3500 - 1:13,000 [4]. Therefore, adding PB V into the equation significantly increases the risk ofperi-operative anaphylaxis [8].

In breast surgery, there is the potential to miss early angioedema because with the surgeon working near to head there is often complete drape coverage of the patient. In this case oedema in the surgical field was only noticed at the point that cardiovascular instability became apparent at 40 minutes after initial dye injection. The use of B-blocker may also have contributed to the severity of hypotension. Delayed reactions are defined as 20 - 30 minutes after blue dye injection [6] but has been reported as late as 67 minutes and are well recognized [9] [10], being particularly difficult to manageif the dye leaches out in a biphasic pattern over time [11].

Clearly there are implications for the anaesthetic staffing of breast lists which may be allocated to relatively junior trainees. As this was the fourth in a series of PBV reactions at our hospital over a 20 month period our institution adopted a temporary policy of arterial line insertion and endotracheal tube placement for all cases requiring PB V. The use of PB V was already part of the safety brief in theatre. Whilst the superiority of Noradrenaline over Adrenaline in promoting cardiovascular stability has been previously described [6] it is not supported in all reports [12]. This is the first report of the potential benefit of continuous veno-venous haemofiltration in mitigating the cardiovascular effects of anaphylaxis due to blue PB V.

It is important to monitor all cases of anaphylaxis for an extended time postoperatively in a high dependency environment as there may be cyclical relapses in the clinical condition [4] [5]. Chronic administration of B-blockers in any patient will make the patient more resistant to cardiovascular resuscitation. In addition any day case patients demonstrating allergic features from any cause should be observed for at least 8 hours prior to discharge. This risk should be borne in mind when pursuing same day discharge programmes in which the patient leaves hospital late in the evening. A recent MHRA guidance [13] endorsed by the Royal College of Surgeons of England, reminds us that all serious allergic reactions should be reported, as was this case, under the "yellow card" scheme. "Patent Bleu V does not carry a licence for marketing and surgeons should have competent personnel and emergency facilities for at least an hour following administration of Patent Bleu V as delayed reactions occur".

\section{Conclusions}

Sentinel Node Biopsy with a dual localization technique using Patent Blue Dye remains the standard of care for clinically node negative breast cancer patients. Although allergic reactions are rare and life-threatening, this ar- 
ticle describes a successful novel approach. Alternatives techniques using fluorescent Indocyanine green [14] and iron grains [15] have been shown to be effective for sentinel node biopsy in breast cancer and may prove a safer and long-term alternative to blue dye.

In the meantime, our experience would prompt the following recommendations: to ensure all theatre personnel are aware of the potential for delayed blue dye reaction and to provide continuous and effective cardiovascular monitoring, visualisation of the patients' face and easy access to vasopressor. If cardiovascular instability persists despite optimal inotropic and respiratory support, the addition of veno-venous haemofiltration may help improve the clinical status of the patient by reducing antigenic load.

\section{Acknowledgements}

The authors appreciate our patients' permission to share this report. This case study was briefly referenced in a summary of four patients reported to Anaesthesia 2011 66(9) 841-2.

\section{Role of the Funding Source}

No funding was received for this study.

\section{References}

[1] National Institute for Health and Clinical Excellence Guideline (2009) Breast Cancer (Early \& Locally Advanced): Diagnosis and Treatment. http://www.nice.org.uk/CG80

[2] Keshtgar, M., Aresti, N. and MacNeil, F (2010) Establishing Axillary Sentinel Node Biopsy SLNB for Early Breast Cancer in the United Kingdom. A Survey of National Training Program. European Journal of Surgical Oncology, 36, 393-398.

[3] Mansel, R.E., Goyal, A., Fallowfield, L. and Newcombe, R.G. (2006) Randomised Multicenter Trial of Sentinel Node Biopsy versus Standard Axillary Treatment in Operable Breast Cancer: The ALMANAC trial. Journal of the National Cancer Institute, 98, 599-609. http://dx.doi.org/10.1093/jnci/djj158

[4] Barthelmes, L., Goyal, A., Newcombe, R.G., McNeill, F. and Mansel, R.E. (2010) Adverse Reactions to Patent Blue V Dye-The NEW START and ALMANAC Experience. European Journal of Surgical Oncology, 36, 399-403.

[5] White, V., Harvey, J.R. and Griffith, C.D.M. (2011) Youssef, Carr MSentinel Node Biopsy in Early Breast Cancer Surgery-Working with the Risks of Vital Blue Dye to Reap the Benefits. European Journal of Surgical Oncology, 37, 101-108.

[6] Quincey, N., Jones, C. and Kissin, M. (2012) Anaphylaxis to Dye during Breast Surgery. Anaesthesia, 67, 80.

[7] Masamat, Y., Shenoy, H., Spiers, V., Hanby, A. and Horgan, K. (2006) Properties and Characteristics of the Dyes Injected to Assist Axillary Sentinel Node Localization in Breast Surgery. European Journal of Surgical Oncology, 32, 381-384.

[8] Laxenaire, M.C. and Mertes, P.M. (2001) Anaphylaxis during Anaesthesia. Results of a Two-Year Survey in France. British Journal of Anaesthesia, 87, 549-558. http://dx.doi.org/10.1093/bja/87.4.549

[9] Hunting, A.S., Nopp, A., Johansson, S.G.O.F., Wilhelmsen, V. and Guttormsen, A.B. (2010) Anaphylaxis to Patent Blue VI Clinical Aspects. Allergy, 45, 117-123.

[10] Lucas, N., Intern, S.B., Laine, P., Nicolie, B. and Fondriner, E. (2010) Anaphylactic Shock Due to Patent Blue; Four Case Report and Review of the Literature. Journal de Gynécologie Obstétrique et Biologie de la Reproduction, 39, 116-120. http://dx.doi.org/10.1016/j.jgyn.2009.10.006

[11] Stark, B.J. and Sullivan, T.J. (1986) Biphasic and Protracted Anaphylaxis. Journal of Allergy and Clinical Immunology, 78, 76. http://dx.doi.org/10.1016/0091-6749(86)90117-X

[12] Lantis, S., Filippakis, G. and Hadjiminas, D.J. (2008) Atypical Anaphylactic Reaction to Patent Blue Dye during Sentinel lymph Node Biopsy for Breast Cancer. Annals of the Royal College of Surgeons of England, 90, 338-339.

[13] MHRA Drug Safety Notice 2012. Safety Information on the Use of Blue Dye Patent Blue V. UK National Pharmacovigilence Expert Advisory Group of the Commission on Human Medicines.

[14] Van der Vorst, J.R., Schaafsma, B.E., Verbeek, F.P., Hutteman, M., Mieog, J.S.D., Lowik, C.W.G.M., Liefers, G.J., Frangioni, J.V., van de Velde, C.J.H. and Vahrmeiger A.L. (2012) Randomised Comparison of Near-Infrared Fluorescence Imaging Using Indocyanine Green and $99^{\mathrm{m}}$ Technetium with or without Patent Blue Dye for the Sentinel Lymph Node Procedure in Breast Cancer Patients. Annals of Surgical Oncology, 19, 4104-4111.

http://dx.doi.org/10.1245/s10434-012-2466-4 
[15] Douek, M., Klasse, J., Monypenny, I., Kothari, A., Zechmeister, K., Brown, D., Wyld, L., Drew, P., Garmo, H., Agbaje, O., Pankhurst, Q., Anning, B., Grootendorst, M., Ten Haken, B., Hall-Creggs, M.A., Purushotham, A. and Pinder, S. (2014) Sentinel Node Biopsy Using a Magnetic Tracer versus Standard Technique: The SentiMAG Multicentre Trial. Annals of Surgical Oncology, 21, 1237-1245. 
Scientific Research Publishing (SCIRP) is one of the largest Open Access journal publishers. It is currently publishing more than 200 open access, online, peer-reviewed journals covering a wide range of academic disciplines. SCIRP serves the worldwide academic communities and contributes to the progress and application of science with its publication.

Other selected journals from SCIRP are listed as below. Submit your manuscript to us via either submit@scirp.org or Online Submission Portal.
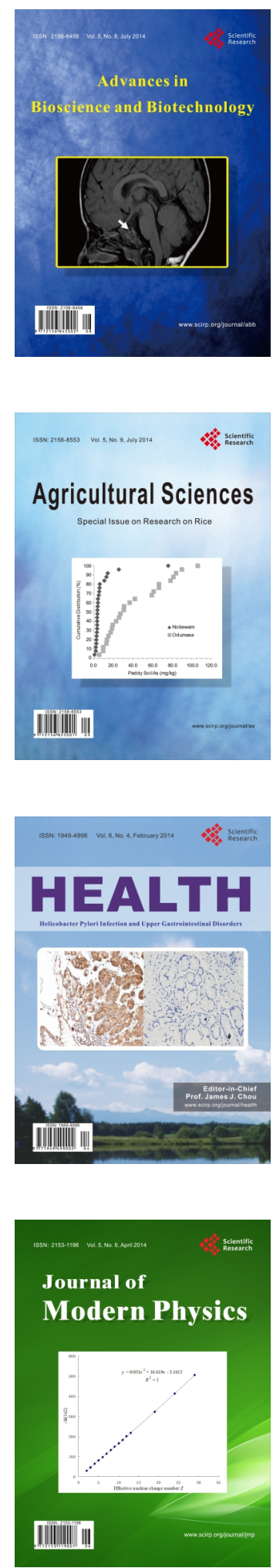
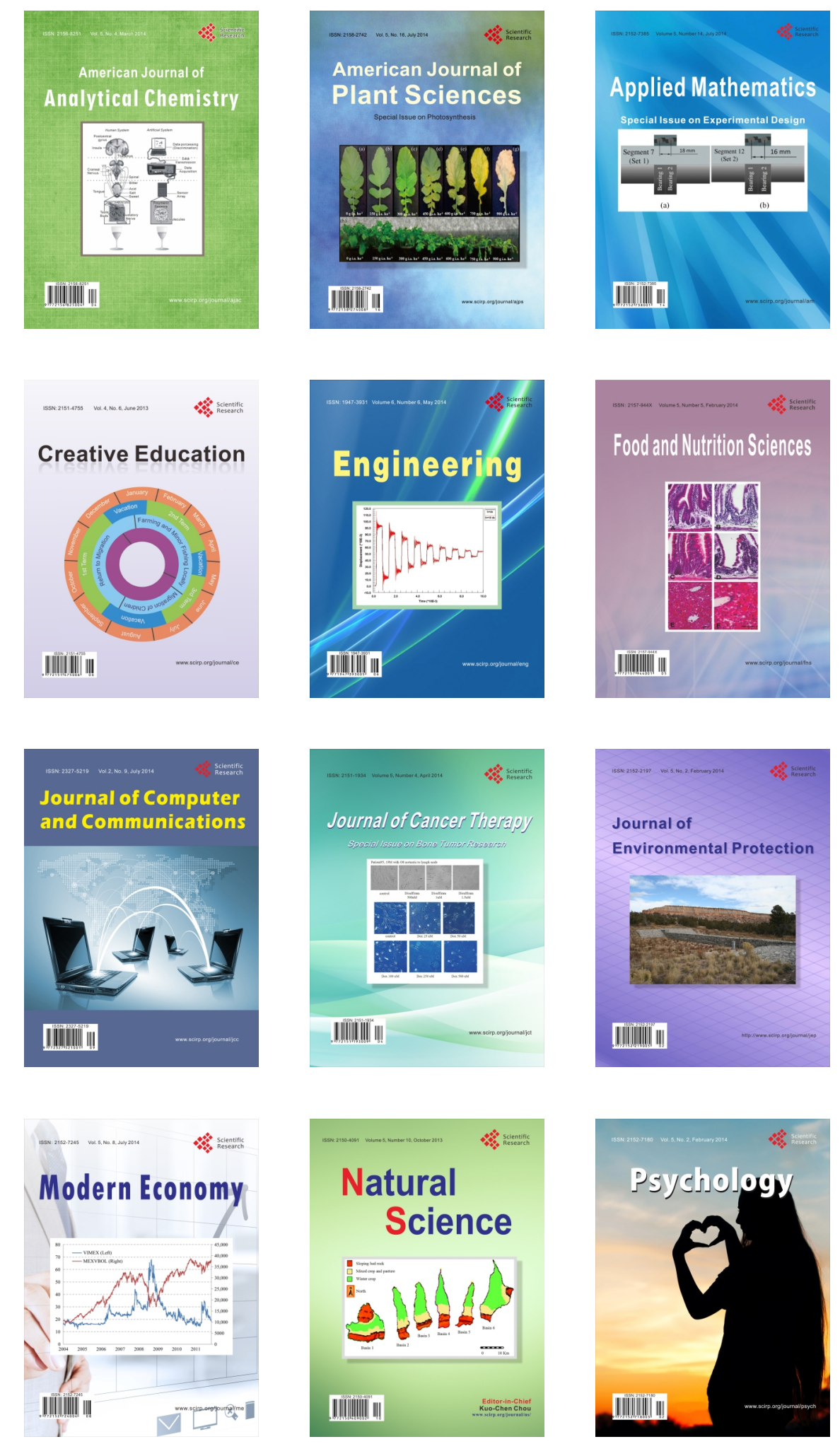